\title{
Caveolin-1 aggravates cigarette smoke extract-induced MUC5AC secretion in human airway epithelial cells
}

\author{
QIAO YU, XI CHEN, XIA FANG, QIONG CHEN and CHENGPING HU \\ Department of Gerontology and Respirology, Xiangya Hospital, \\ Central South University, Changsha, Hunan 410008, P.R. China
}

Received December 29, 2014; Accepted March 4, 2015

DOI: $10.3892 / \mathrm{ijmm} .2015 .2133$

\begin{abstract}
Airway mucus hypersecretion is a major pathological characteristic of chronic obstructive pulmonary disease (COPD), and cigarette smoke is highly implicated in mucus secretion and the development of COPD. Cigarette smoke reportedly induces mucin overproduction through the epidermal growth factor receptor (EGFR) in the airway epithelium; however, the underlying mechanisms responsible for the activation of EGFR remain unknown. Caveolin-1, a component protein in the cytomembrane, reportedly regulates airway inflammation and lung injury. In this study, we aimed to determine whether caveolin-1 modulates mucin hyperproduction induced by cigarette smoke. Our results revealed that cigarette smoke extract (CSE) significantly increased MUC5AC production, as well as the levels of phosphorylated EGFR (p-EGFR) and phosphorylated Akt (p-Akt) in human bronchial epithelial cells (16HBE cells), as shown by ELISA, RT-PCR and western blot analysis. These effects were prevented by treatment with EGFR inhibitor (AG1478) and phosphatidylinostol3-kinase (PI3K) inhibitor (LY294002). We also found that the overexpression of caveolin-1 enhanced the expression of MUC5AC, p-EGFR and p-Akt induced by CSE. Conversely, the downregulation of caveolin-1 by siRNA against caveolin-1 inhibited the expression of MUC5AC, p-EGFR and p-Akt. Taken together, our data suggest that caveolin-1 enhances CSE-induced MUC5AC hypersecretion through the EGFR/ PI3K/Akt signaling pathway.
\end{abstract}

\section{Introduction}

Mucus secretion functions as a guard and barrier for the airway epithelium. An appropriate amount of mucus secretion protects

Correspondence to: Dr Qiong Chen, Department of Gerontology, Xiangya Hospital, Central South University, 87 Xiangya Road, Changsha, Hunan 410008, P.R. China

E-mail: qiongchen2013@gmail.com

Key words: MUC5AC, cigarette smoke extract, caveolin-1, epidermal growth factor receptor, phosphatidylinostol-3-kinase, Akt the airway epithelium from dust particles, smoke, bacteria and viruses, and ciliary movement clears these irritants from the airways $(1,2)$. However, the chronic and hypersecretion of mucus can induce severe airway obstruction and repeated airway infection. It can contribute to the morbidity and mortality of chronic inflammatory pulmonary diseases, particularly chronic obstructive pulmonary disease (COPD) (3-5). Mucins are the main secretory components of mucus $(6,7)$. To date, 20 mucin genes can be found to be expressed in the airways. MUC5AC appears to be the most prominent phenotype, particularly in the pathological state $(6,7)$.

Cigarette smoke (CS) reportedly represents the first risk of developing COPD, and the mortality rate for COPD has been shown to be at least 7-fold higher in smokers than in non-smokers $(8,9)$. Repeated irritation from smoke destroys mucociliary clearance, amplifies mucus production and results in mucus hypersecretion, promoting the development of COPD $(10,11)$. Previous studies have implicated the epidermal growth factor receptor (EGFR) in the response of bronchial epithelial cells to cigarette smoke extract (CSE), including the reduction in epithelial integrity and mucus hypersecretion (12-14). The mechanisms responsible for the secretion of MUC5AC induced by EGFR have been extensively investigated. Studies have demonstrated that CS induces the activation of EGFR, then triggers phosphatidylinostol-3-kinase (PI3K)/Akt and mitogen-activated protein kinase (MAPK) signaling, and finally increases MUC5AC gene expression and secretion (15-17). However, the mechanisms responsible for the activation of EGFR remain unclear.

Caveolae are 50-100 nm flask-shaped invaginations of the plasma membrane (18). Caveolae have been implicated in numerous biological functions, including signal transduction, cellular metabolism, cholesterol homeostasis and tumor suppression (19). The structural proteins required for caveolae are caveolins, including caveolin-1, caveolin-2 and caveolin-3 (20). As previously demonstrated, caveolin-1 is a principal component of caveolar membranes in many cell types, including airway epithelial cells, and it plays physiological roles in regulating signaling molecules within caveolar membranes (21). These signaling molecules include heterotrimeric G proteins, the Src-family, non-receptor tyrosine kinases, endothelial nitric oxide synthase (eNOS) and p42/44 MAPK (21-23). Researchers have found that caveolin-1 interacts with these signaling molecules through the caveolin-1 scaffolding domain (CSD) (21). For example, EGFR 
mostly gathers in the caveolae and caveolin-1 regulates the activation of EGFR and signaling from EGFR to the nucleus, resulting in reduced cell growth and increased apoptosis (24-26).

Previous studies have discovered the potential roles of caveolin-1 in lung inflammation and injury $(27,28)$. Caveolin-1 can reportedly regulate the development of lung injury by modulating the acute inflammatory response, capillary leakage and pulmonary edema. Researchers have also found that the deletion of caveolin-1 increases the production of the pro-inflammatory cytokines, interleukin-6 (IL-6) and tumor necrosis factor- $\alpha$ (TNF- $\alpha$ ), induced by lipopolysaccharides $(28,29)$. These studies, combined with the close association between MUC5AC secretion and airway inflammation, led us to hypothesize that caveolin-1 may be an important regulator involved in CS-induced MUC5AC production in lung epithelial cells. To examine this hypothesis, we employed gainand loss-function approaches to assess the effects of caveolin-1 on the secretion of MUC5AC stimulated by CSE, as well as the underlying mechanisms.

\section{Materials and methods}

Cells, reagents and antibodies. Human bronchial epithelial cells (16HBE cells) were purchased from Fuxiang Biotechnology Co., Ltd. (Shanghai, China). Fetal bovine serum (FBS), trypsin, Roswell Park Memorial Institute (RPMI)-1640 medium, and Opti-MEM were obtained from Gibco (Carpinteria, CA, USA). Lipofectamine 2000, primers for polymerase chain reaction (PCR), TRIzol reagent and the PCR kit were purchased from Invitrogen (Carlsbad, CA, USA). TransIT-TKO reagents were purchased from Mirus Bio Corp. (Madison, WI, USA). Rabbit anti-human caveolin-1 (\#3238), rabbit anti-human EGFR (\#2232), rabbit anti-human Akt (\#9272), rabbit anti-human phosphorylated Akt (p-Akt; \#9271) and mouse anti-human phosphorylated EGFR (p-EGFR; \#2236) antibodies, as well as LY294002 (a specific inhibitor of PI3K) were purchased from Cell Signaling Technology (Beverly, MA, USA). Mouse anti-human $\beta$-actin antibody, dimethyl sulfoxide (DMSO) and cocktail were purchased from Sigma (St. Louis, MO, USA). Horseradish peroxidase (HRP)-conjugated polyclonal antimouse and anti-rabbit secondary antibodies were purchased from Jackson Immunoresearch, Inc. (West Grove, PA, USA). AG1478, a specific inhibitor of EGFR was purchased from Enzo Life Sciences (Farmingdale, NY, USA). Caveolin-1-expressing plasmid and small interfering RNA (siRNA) were designed by GeneChem Co. (Shanghai, China). Phosphatase inhibitors were purchased from Roche (Basel, Switzerland). The enzyme-linked immunosorbent assay (ELISA) for the detection of MUC5AC was obtained from Cusabio Biotech Co. (Wuhan, China).

Preparation of CSE. CSE was prepared as previously described (30-32). CSE (100\%) was prepared by bubbling smoke from 2 cigarettes in $10 \mathrm{ml}$ of serum-free RPMI-1640 medium at a rate of half a cigarette/min. The $\mathrm{pH}$ of the CSE was adjusted to 7.4 and CSE was sterile-filtered through a $0.22-\mu \mathrm{M}$ filter. The CSE was always freshly prepared on the day of the experiment.

Cell culture and treatment. The $16 \mathrm{HBE}$ cells were propagated in RPMI-1640 supplemented with 10\% FBS, 100 U/ml penicillin and $100 \mu \mathrm{g} / \mathrm{ml}$ streptomycin in a $37^{\circ} \mathrm{C} 5 \% \mathrm{CO}_{2}$ incubator. The cells were replenished with fresh medium every 2-3 days. Before additional treatments, the cells were plated in $60 \times 60 \mathrm{~mm}$ culture dishes at a density of $2 \times 10^{6}$ cells $/ \mathrm{ml}$ and were cultured in a $37^{\circ} \mathrm{C}$ $5 \% \mathrm{CO}_{2}$ incubator. Following serum starvation for $12 \mathrm{~h}$, the cells were treated with CSE at various concentrations $(0,2,5,10,15$ and $20 \%)$ for $24 \mathrm{~h}$ or $10 \% \mathrm{CSE}$ for different periods of time $(0$, 6, 12, 24 and 48 h). Before the subsequent experiments, MTT assay was used to evaluate the viability of the $16 \mathrm{HBE}$ cells. The following experiments were performed using a culture of $>80 \%$ viable cells.

To investigate the signaling cascade from the CSE-induced secretion of MUC5AC, the cells were pre-treated with $10 \mu \mathrm{M}$ AG1478 (EGFR inhibitor) for 30 min or with $50 \mu \mathrm{M} \mathrm{LY} 294002$ (PI3K inhibitor) for $1 \mathrm{~h}(33,34)$. Following $24 \mathrm{~h}$ of incubation in a culture medium containing $10 \% \mathrm{CSE}$, the cells and culture supernatants were harvested for further analysis.

In order to induce the overexpression or downregulation of caveoln-1, the 16HBE cells were transiently transfected with caveolin-1-expressing plasmid and empty vector plasmid using Lipofectamine 2000 or caveolin-1 siRNA plasmid and siRNA control plasmid using TransIT-TKO reagent. Following incubation with the plasmids for 4-6 h, the cells were treated with CSE (10\%) for $24 \mathrm{~h}$.

ELISA for MUC5AC in the cell supernatant. The cell culture supernatants were collected and used to assay the total protein concentration. MUC5AC protein epxression was measured following the instructions provided with the ELISA kit.

Reverse transcription-polymerase chain reaction analysis $(R T-P C R)$. Total RNA was extracted from the $16 \mathrm{HBE}$ cells in each group using TRIzol reagent. The extraction was verified by electrophoresis on a $1.0 \%$ agarose gel and an absorbance (A260/280) value of 1.8-2.0. Reverse transcription for complementary DNA was performed using an RT-PCR kit. The PCR primers for MUC5AC were 5'-AACTGCAGCTGGACA GTGTG-3' (forward) and 5'-TGCAGATCTGGGTCTC ACAG-3' (reverse); and those for $\beta$-actin were 5'-GGGCA CGAAGGCTCATCATT-3' (forward) and 5'-AGCGAGCATC CCCCAAAGTT-3' (reverse). The PCR reactions were carried out as follows: a pre-denaturing at $94^{\circ} \mathrm{C}$ for $5 \mathrm{~min}$, followed by 28 cycles of denaturation at $94^{\circ} \mathrm{C}$ for $45 \mathrm{sec}$, annealing at $58^{\circ} \mathrm{C}$ for $40 \mathrm{sec}$, and extension at $72^{\circ} \mathrm{C}$ for $45 \mathrm{sec}$. PCR products were separated by electrophoresis through $1 \%$ agarose gel containing ethidium bromide, and the signal intensity was analyzed using Quantity One software.

Western blot analysis. The cells were lysed in a RIPA lysis buffer with protease inhibitor and phosphatase inhibitor. Equal amounts of cell lysate from the protein samples were resolved by 10 and $12 \%$ sodium dodecyl sulfate-polyacrylamide gel electrophoresis (SDS-PAGE) and transferred onto polyvinylidene difluoride (PVDF) membranes. These membranes were incubated with $5 \%$ skimmed milk or $5 \%$ BSA in PBST at room temperature for $1 \mathrm{~h}$ and exposed to specific primary antibodies against caveolin-1, EGFR, p-EGFR, Akt and p-Akt (at $1: 1,000$ ) overnight at $4^{\circ} \mathrm{C}$. This was then followed by incubation with HRP-conjugated goat anti-mouse and anti-rabbit secondary antibodies (at 1:10,000) for $1 \mathrm{~h}$ at room temperature. 


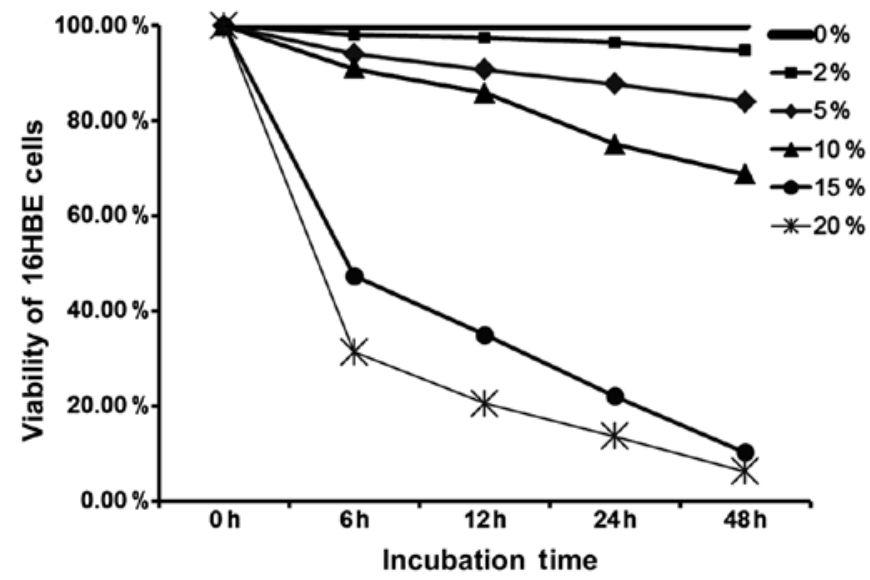

Figure 1. Viability of human bronchial epithelial cells (16HBE cells) following exposure to cigarette smoke extract (CSE). The 16HBE cells were exposed to $0,2,5,10,15$ and $20 \%$ CSE for $0,6,12,24$ and $48 \mathrm{~h}$, and MTT assay was used to determine the viability of the $16 \mathrm{HBE}$ cells.

The blots were visualized by enhanced chemiluminescence. The intensity of each band was measured using Quantity One software. The relative protein expression level was determined by normalization to that of $\beta$-actin.

Statistical analysis. Data were analyzed using Pearson's correlation co-efficient with SPSS 17.0 software (SPSS, Inc., Chicago, IL, USA) and are presented as the means \pm standard deviation. The data were also analyzed using the Student's t-test or one-way analysis of variance followed by the Tukey's test where appropriate. P-values $<0.05$ were considered to indicate statistically significant differences.

\section{Results}

Cell cytotoxicity. Cell cytotoxicity was detected by MTT assay. CSE exerted cytotoxic effects on the 16HBE cells in a time- and concentration-dependent manner. A low concentration and short time treatment of CSE had no effect on cell viability, and the viability of the $16 \mathrm{HBE}$ cells decreased with the increasing CSE concentration and the increase in the treatment duration (Fig. 1).

Effect of CSE on MUC5AC synthesis. We selected $24 \mathrm{~h}$ as the best incubation time due to the MTT results and the results from previous studies $(33,35)$. The synthesis of MUC5AC mRNA and protein exhibited a dose-dependent increase in response to treatment with CSE for $24 \mathrm{~h}$. The mRNA expression level of MUC5AC was increased by approximately 2.5-, 4.3- and 7.5-fold following incubation with 2, 5 and $10 \%$ CSE, respectively $(\mathrm{p}<0.05)$. Stimulation with 2,5 and $10 \%$ CSE induced a 1.9-, 3.0- and 4.3-fold increase, respectively in the protein expression of MUC5AC in the culture supernatant $(\mathrm{p}<0.05)$. The cells exposed to CSE showed an increase in the mRNA and protein expression of MUC5AC in a concentration-dependent manner (Fig. 2).

EGFR and PI3K/Akt signaling pathways mediate CSE inducedMUC5AC expression. Previous studies have revealed a close

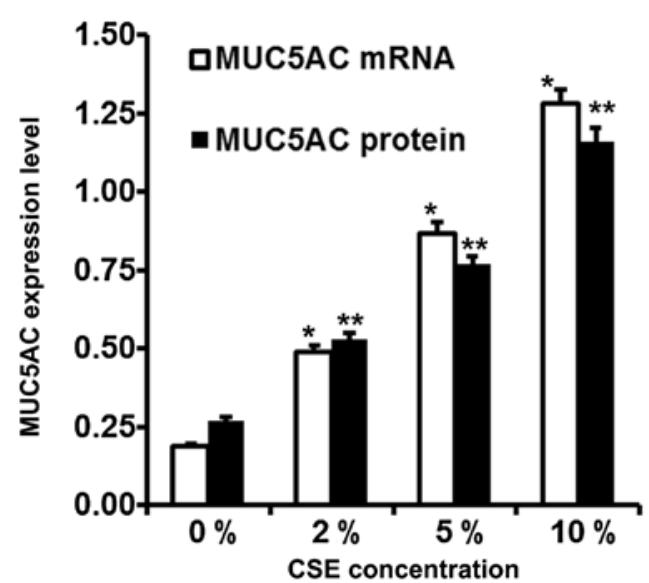

Figure 2. Changes in MUC5AC expression indcued by cigarette smoke extract (CSE) in human bronchial epithelial cells (16HBE cells). 16HBE cells were treated with various concentrations of cigarette smoke extract (CSE) for $24 \mathrm{~h}$. mRNA expression was assessed by RT-PCR and normalized to $\beta$-actin. Protein production was detected using a MUC5AC enzyme-linked immunosorbent assay (ELISA) kit. " $\mathrm{p}<0.05$ and ${ }^{* *} \mathrm{p}<0.05$, compared with the control group. The data are representative of 3 separate experiments.

association between CSE and EGFR activation (12-14). Thus, we examined whether the EGFR and PI3K/Akt signaling pathways are essential for the CSE-induced secretion of MUC5AC. EGFR inhibitor (AG1478) and PI3K inhibitor (LY294002) were used to treat the 16HBE cells prior to CSE stimulation. Exposure of the $16 \mathrm{HBE}$ cells to CSE led to a significant increase in the levels of p-EGFR ( $p<0.05$ vs. control group), and the activation of EGFR by CSE was partially inhibited by AG1478 ( $<<0.05$ vs. CSE group). The levels of $\mathrm{p}$-Akt increased following CSE stimulation ( $<<0.05$ vs. control group), and LY294002 markedly attenuated Akt phosphorylation, but did not affect EGFR phosphorylation ( $\mathrm{p}<0.05$ vs CSE group, $\mathrm{p}>0.05$ vs. CSE group) (Fig. 3). Pre-treatment with AG1478 or LY294002 markedly abrogated the upregulation of MUC5AC mRNA expression and protein production induced by CSE (Fig. 4).

Regulation of CSE-mediated MUC5AC expression by caveolin-1 in 16HBE cells. To determine whether the CSE-induced MUC5AC expression is associated with caveolin-1, we either enforced the expression of caveolin-1 in the 16HBE cells by transfection with caveolin-1-expressing plasmid, or downregulated its expression by transfection with caveolin-1 siRNA. An approximately $100 \%$ elevation in MUC5AC mRNA and a $50 \%$ elevation in MUC5AC protein levels were achieved by the delivery of caveolin-1-expression plasmids compared to transfection with the control vector. Conversely, the knockdown of caveolin-1 by siRNA resulted in an approximatley 1 -fold reduction in the MUC5AC mRNA and protein expression levels compared to the cells treated with the siRNA control (Fig. 5). These results suggest that caveolin-1 acts as a positive regulator of the hypersecretion of MUC5AC by CSE; caveolin-1 had no effect on MUC5AC secretion in the absence of CSE (Fig. 5).

EGFR/PI3K/Akt signaling pathway is involved in the effects of caveolin-1 on CSE-induced MUC5AC expression. As verified above, CSE induced MUC5AC expression through 

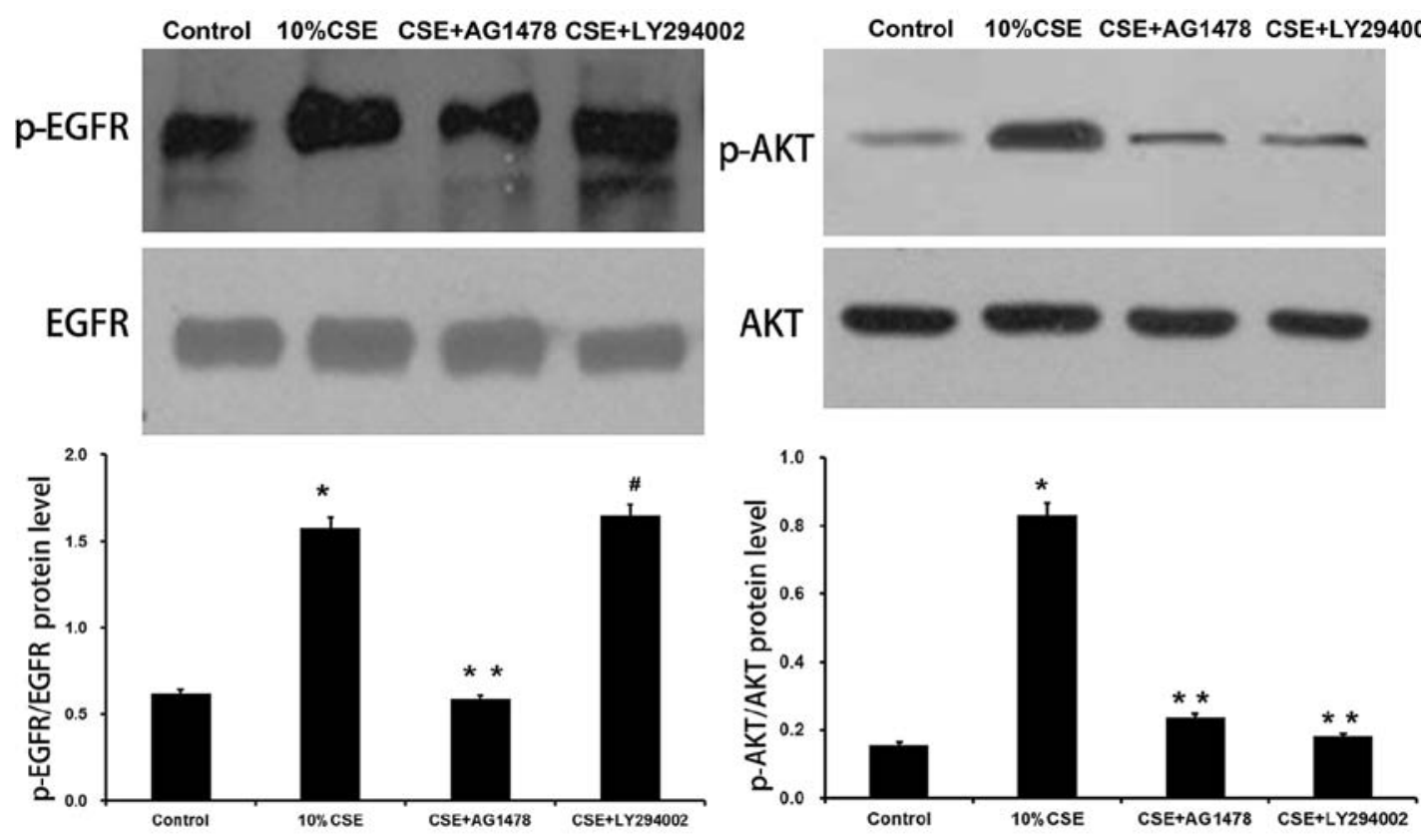

Figure 3. Activation of epidermal growth factor receptor (EGFR)-mediated phosphatidylinostol-3-kinase (PI3K)/Akt signaling pathway by cigarette smoke extract (CSE) in human bronchial epithelial cells (16HBE cells). Cells were divided into 4 different treated groups. 16HBE cells were pre-treated with $10 \mu \mathrm{M}$ AG1478 for $1 \mathrm{~h}$, or $50 \mu \mathrm{M} \mathrm{LY} 294002$ for $1 \mathrm{~h}$, and then stimulated with CSE for 24h. EGFR and Akt phosphorylation levels were assessed by western blot analysis. "p<0.05, compared to the untreated control group; ${ }^{* *} \mathrm{p}<0.05$ and ${ }^{\#} \mathrm{p}>0.05$, compared with the CSE-treated group. Results are representative of 3 independent experiments.

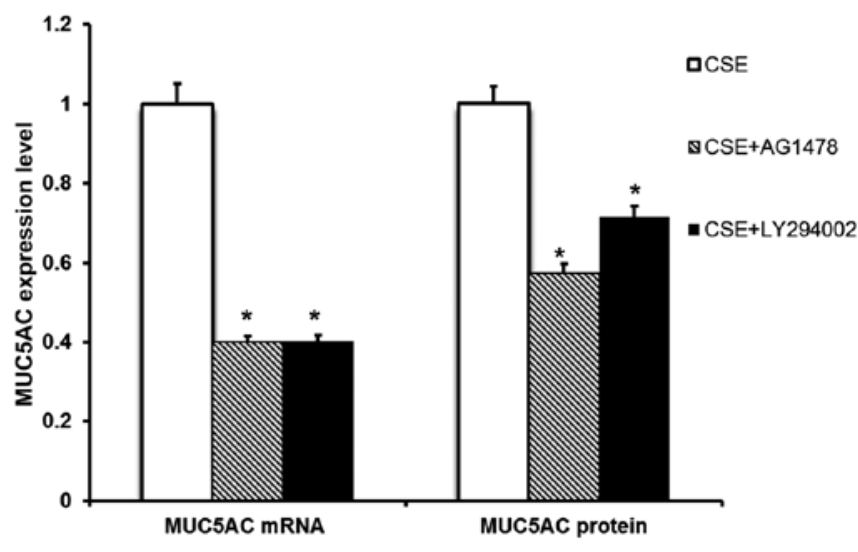

Figure 4. Regulation of cigarette smoke extract (CSE)-induced MUC5AC expression by epidermal growth factor receptor (EGFR) and phosphatidylinostol-3-kinase (PI3K). Cells were treated with AG1478 $(10 \mu \mathrm{M})$ for $1 \mathrm{~h}$ or LY294002 $(50 \mu \mathrm{M})$ for $1 \mathrm{~h}$, and then incubated with CSE for $24 \mathrm{~h}$. ${ }^{\mathrm{p}}<0.05$, compared with the CSE-treated group. Data represent 3 independent experiments.

the EGFR/PI3K/Akt signaling pathway and caveolin-1 enhanced the CSE-mediated MUC5AC expression. Western blot analysis revealed that the overexpression of caveolin-1 induced in the 16HBE cells by caveolin-1-expressing plasmid caused a marked increase in EGFR and Akt phosphorylation. Conversely, transfection with caveolin-1 siRNA prior to incubation with CSE significantly decreased the levels of p-EGFR and p-Akt (Fig. 6). Furthermore, pre-treatment with AG1478 or LY294002 markedly abrogated the upregulation of MUC5AC expression induced by CSE through caveolin-1 in the 16HBE cells (Fig. 7).

\section{Discussion}

Caveolin-1, a 21-24 $\mathrm{kDa}$ cytomembrane protein, is the major resident scaffolding protein constituent of caveolae that participates in vesicular trafficking and signal transduction $(21,36)$. Caveolin-1 participates in several biological processes, including cell growth, apoptosis and cell proliferation (18). A recent study indicated that caveolin-1 also plays a role in the development of lung inflammation (28). Yuan et al found that caveolin-1 knockout mice exhibited an increase in inflammation and in the levels of superoxide in the lungs, and presented with aggravated severe lung injury after Pseudomonas aeruginosa infection (29). In alveolar and peritoneal macrophages, researchers have shown that the downregulation of caveolin-1 increases the LPS-induced production of the pro-inflammatory cytokines, TNF- $\alpha$ and IL-6. By contrast, it decreased anti-inflammatory cytokine IL-10 production (37). However, studies have produced conflicting results on whether caveolin-1 attenuates lung inflammation. The overexpression of caveolin-1 was shown to aggravate the alveolar type-I cell injury induced by LPS in the study by Lv et al (32). In another study using caveolin-1 null mice, Hu et al demonstrated that in polymorphonuclear neutrophils (PMN) from these mice, PMN activation, adhesion, as well as PMN activation-induced lung inflammation and vascular injury were reduced (38). These data indicate that caveolin-1 is involved in lung inflammation, although whether its role is promotional or suppressive remains controversial. As is already known, inflammatory reactions are essential processes in MUC5AC secretion, and as mentioned above, caveolin-1 is an important regulator of inflammation. It can thus be hypothesized that caveolin-1 may be associated with MUC5AC secretion. 


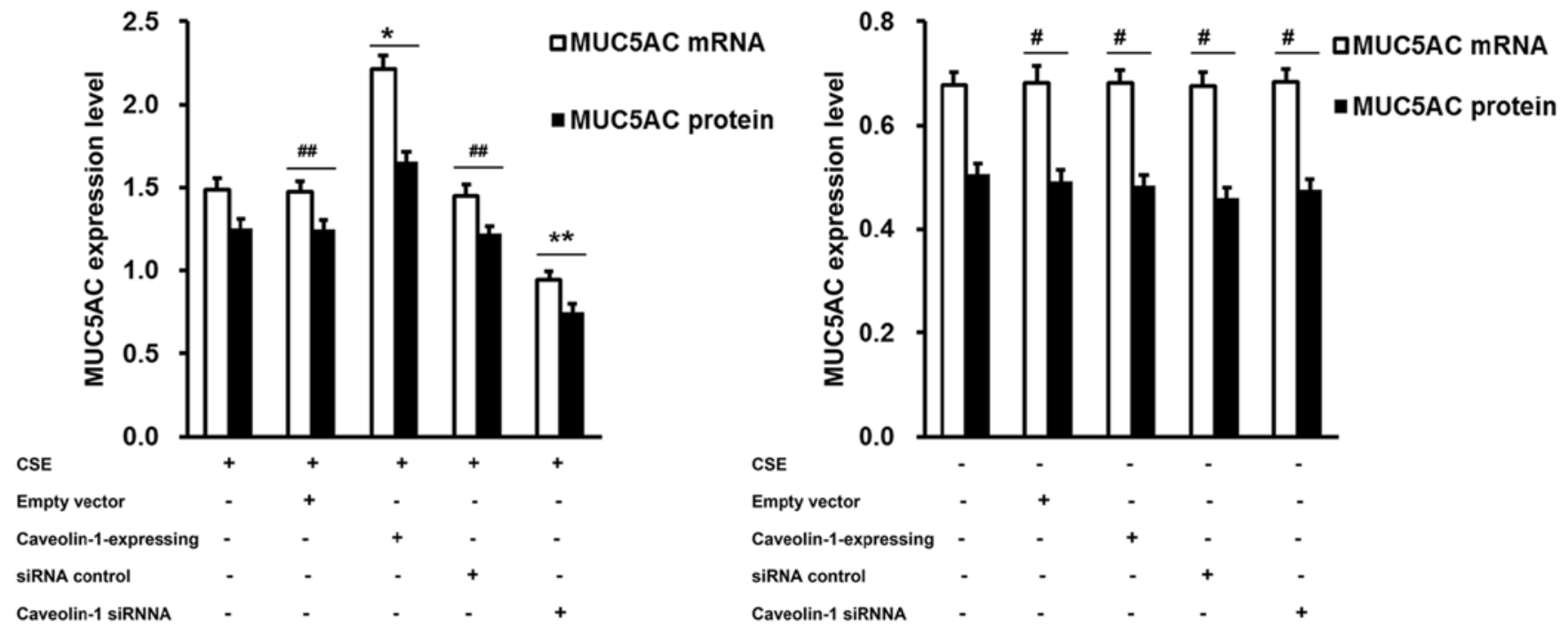

Figure 5. Effect of caveolin-1 on the MUC5AC expression. For inducing the overexpression or knockdown of caveolin-1, human bronchial epithelial cells (16HBE cells) were transiently transfected with caveolin-1-expressing plasmids or caveolin-1 siRNA. At 4-6 h post-transfection, the cells were treated with $10 \%$ cigarette smoke extract (CSE) for $24 \mathrm{~h}$. " $\mathrm{p}<0.05$ vs. group transfected with empty vector; ${ }^{* *} \mathrm{p}<0.05 \mathrm{vs}$. siRNA control group; ${ }^{\# \#} \mathrm{p}>0.05$ vs. CSE only-treated group; ${ }^{\#}>0.05$ vs. untreated group. Data represent 3 independent experiments.
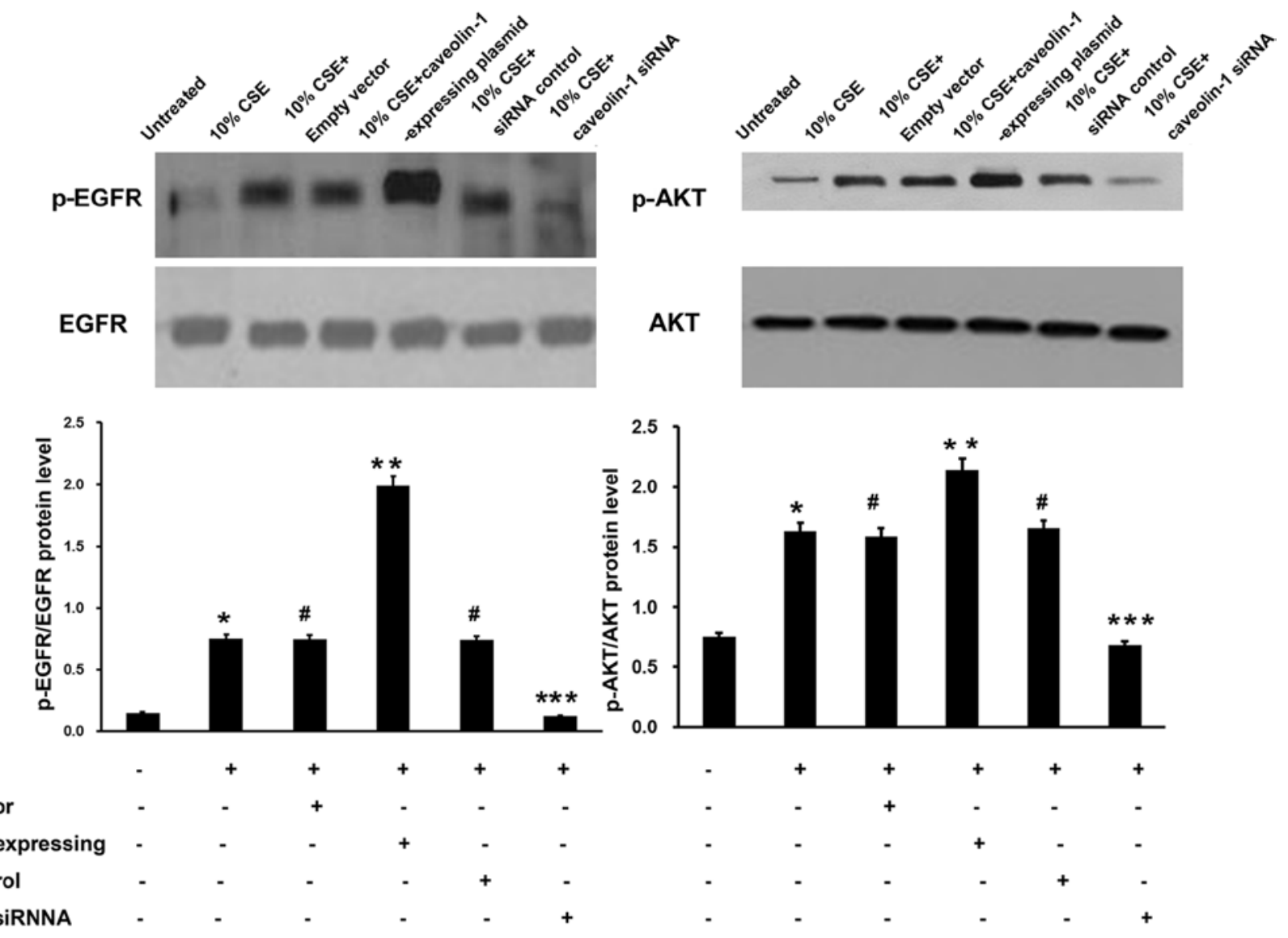

AKT

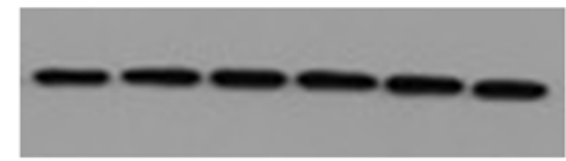

Figure 6. Regulation of epidermal growth factor receptor (EGFR)/phosphatidylinostol-3-kinase (PI3K)/Akt signaling pathway by caveolin-1. Cells were divided into 6 different treatment groups. " $\mathrm{p}<0.05$ vs. untreated group; ${ }^{*} \mathrm{p}<0.05$ vs. cigarette smoke extract (CSE)-treated group; ${ }^{* *} \mathrm{p}<0.05$ vs. the group transfected with the empty vector; ${ }^{* * * *} \mathrm{p}<0.05$ vs. siRNA control group. Data represent 3 independent experiments.

In this study, we established an in vitro model of MUC5AC hypersecretion induced by CSE using HBE cells. We used two independent methods to determine the role of caveolin-1 in MUC5AC secretion. A gain-of-function experiment using a 

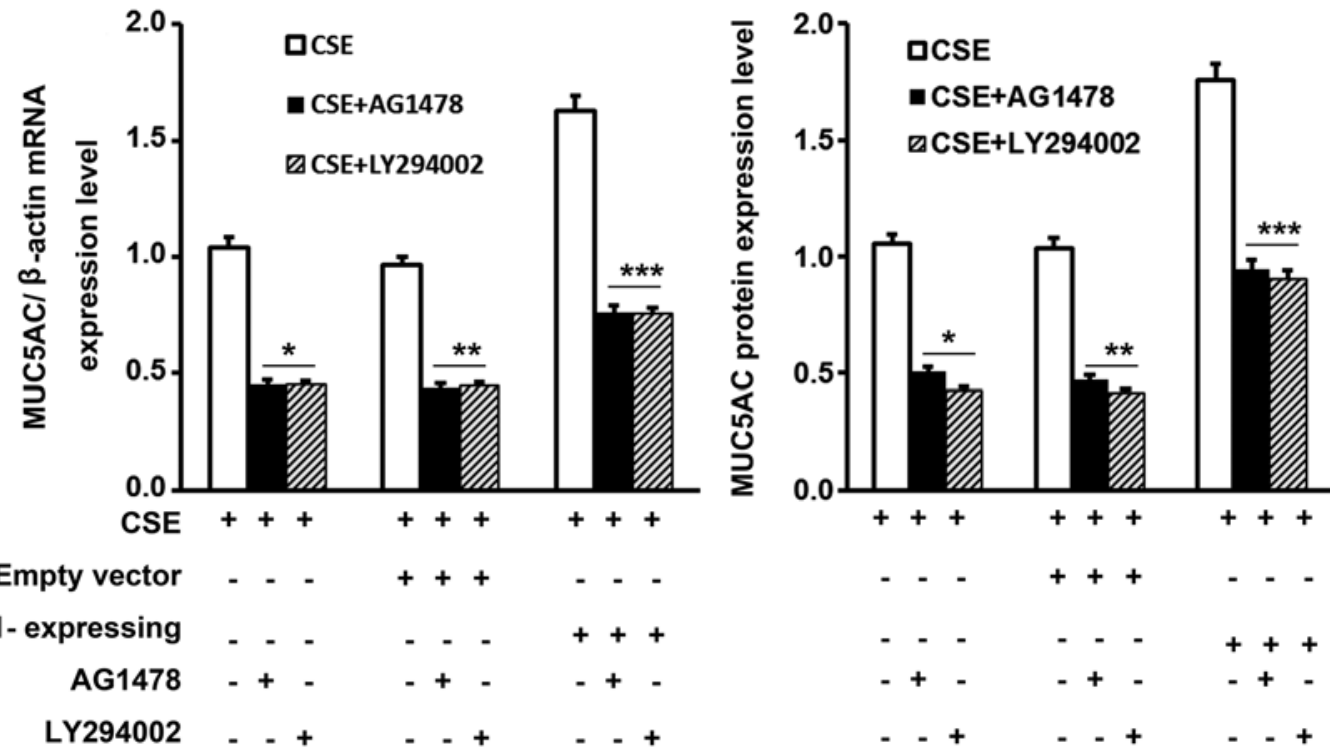

Figure 7. Involvement of the epidermal growth factor receptor (EGFR) and phosphatidylinostol-3-kinase (PI3K)/Akt pathway in the induction of MUC5AC expression induced by cigarette smoke extract (CSE) enhanced by caveolin- 1 overespression. The cells were divided into 9 different treatment groups. ${ }^{*}<<0.05$, compared with the CSE-treated group; ${ }^{* *} \mathrm{p}<0.05$, compared with CSE + empty vector-treated group; ${ }^{* * *} \mathrm{p}<0.05$, compared with CSE + caveolin-1 expressing plasmid-treated group. Data represent 3 independent experiments.

caveolin-1-expressing plasmid transfection demonstrated that the overexpression of caveolin-1 in the 16HBE cells increased the CS-induced production of MUC5AC. A loss-of-function experiment using transfection with caveolin-1 siRNA decreased the secretion of MUC5AC induced by CS. Our results demonstrated that caveolin-1 promoted the CS-induced secretion of MUC5AC.

The biological significance of caveolin-1 is dependent on its interaction with signaling molecules and regulating their activation $(18,23,36)$. Previous studies have indicated that EGFR is concentrated in the caveolae, and that caveolin-1 modulates EGFR activation, leading to its complex involvement in diseases $(39,40)$.

CS is considered to be a significant etiology of mucin hypersecretion. One way that CS exerts its biological effects is by binding to EGFR, then leading to the activation of a cascade of signaling pathways (41). Several studies have raised the possibility that CS causes EGFR activation by increasing the availability of soluble EGFR ligands [e.g., transforming growth factor-bilamphiregulin], which then bind to and activate EGFR in airway epithelial cells (42). In a previous study, exposure to CS upregulated EGFR mRNA expression and induced EGFR-specific tyrosine phosphorylation, resulting in the upregulation of MUC5AC mRNA and protein expression. These effects were inhibited by selective EGFR tyrosine kinase inhibitors (12). The activation of EGFR promotes downstream signaling, such as PIK/Akt, p38 MAPK and ERK1/2 (41). The EGFR/PI3K/Akt pathway is a key step in MUC5AC production activated by a number of stimuli, including CS $(34,43,44)$. We thus hypothesized that EGFR/PI3K/Akt signaling cascades are possible attractive target candidates for caveolin-1.

In this study, we found that the overexpression of caveolin-1 increased the levels of p-EGFR and p-Akt induced by CS compared with the downregulation of caveolin-1 by siRNA. EGFR inhibitor (AG1478) blocked the effects of CS on the levels of p-EGFR and p-Akt, and MUC5AC production. PI3K inhibitor (LY294002) attenuated the increase in the levels of p-Akt and MUC5AC production, but did not affect p-EGFR expression. These results indicate that caveolin-1 affects CS-induced MUC5AC production through EGFR phosphorylation and the activation of the EGFR/PI3K/ Akt signaling pathway. Furthermore, we discovered that in the absence of CSE, caveolin-1 did not regulate MUC5AC secretion, which suggests that caveolin-1 has no effect on the basic secretion of MUC5AC, but on CS-induced MUC5AC secretion.

Our data, as well as previously published data, suggest that caveolin-1 interacts with EGFR to promote its activation and downstream signaling. The study by Wang et al asserted that the hypoxia-induced factor (HIF)-dependent upregulation of caveolin-1 enhanced the phosphorylation of EGFR and increased the proliferation, migration and invasion capacities of renal cell carcinoma cells (45). The disruption of caveolae by Filipin III and M $\beta C D$ has been shown to significantly attenuate the endothelin-1-induced phosphorylation of EGFR in mesangial cells in the study by Hua et al (46). Intriguingly, caveolin-1 was initially considered to be a negative regulator of signaling molecules (21). The overexpression of caveolin-1 in MCF-7 cells has been shown to decrease the phosphorylation of EGFR in breast cancer (47). Another study demonstrated that infection with adenovirus encoding caveolin-1 significantly inhibited angiotensin II-induced EGFR activation, hypertrophy and the migration of vascular smooth muscle cells (VSMCs). Methyl- $\beta$-cyclodextrin (Mclod, a disrupter of caveolae structure, stimulated EGFR activation in VSMCs (40). In addition, Mattson et al demonstrated that the overexpression 
of caveolin-1 had no effect on the phosphorylation of EGFR in fatty cells (48). To date, evidence suggests that the multiple roles of caveolin-1 in EGFR activation may be dependent on the types of cell and irritants $(23,28,49)$. Futher studies are required to clarify the exact mechanisms involved.

In conclusion, to the best of our knowledge, we demonstrate for the first time that caveolin-1 plays a promoting role in CS-induced MUC5AC secretion in 16HBE cells. We also provide evidence that the effects of caveolin-1 involve the EGFR/PI3K/Akt signaling pathway. It can thus be hypothesized that the downregulation of caveolin-1 protects against the mucus hypersecretion induced by CSE. Caveolin-1 may be a potential target for the treatment of CS-induced mucus hypersecretion in COPD.

\section{References}

1. Voynow JA and Rubin BK: Mucins, mucus, and sputum. Chest 135: 505-512, 2009

2. Adler KB, Tuvim MJ and Dickey BF: Regulated mucin secretion from airway epithelial cells. Front Endocrinol 4: 129, 2013.

3. Turner J and Jones CE: Regulation of mucin expression in respiratory diseases. Biochem Soc Trans 37: 877-881, 2009.

4. Cerveri I and Brusasco V: Revisited role for mucus hypersecretion in the pathogenesis of COPD. Eur Respir Rev 19: 109-112, 2010.

5. Curran DR and Cohn L: Advances in mucous cell metaplasia: A plug for mucus as a therapeutic focus in chronic airway disease. Am J Respir Cell Mol Biol 42: 268-275, 2010.

6. Rose MC and Voynow JA: Respiratory tract mucin genes and mucin glycoproteins in health and disease. Physiol Rev 86 : 245-278, 2006

7. Evans CM and Koo JS: Airway mucus: The good, the bad, the sticky. Pharmacol Ther 121: 332-348, 2009.

8. Halbert RJ, Natoli JL, Gano A, Badamgarav E, Buist AS and Mannino DM: Global burden of COPD: Systematic review and meta-analysis. Eur Respir J 28: 523-532, 2006.

9. Kinnula VL, VasankariT, KontulaE,Sovijarvi A,Saynajakangas O and Pietinalho A: The 10-year COPD Programme in Finland: Effects on quality of diagnosis, smoking, prevalence, hospital admissions and mortality. Prim Care Respir J 20: 178-183, 2011.

10. Yoshida T and Tuder RM: Pathobiology of cigarette smokeinduced chronic obstructive pulmonary disease. Physiol Rev 87: 1047-1082, 2007

11. Tamimi A, Serdarevic D and Hanania NA: The effects of cigarette smoke on airway inflammation in asthma and COPD Therapeutic implications. Respir Med 106: 319-328, 2012.

12. Takeyama K, Jung B, Shim JJ, et al: Activation of epidermal growth factor receptors is responsible for mucin synthesis induced by cigarette smoke. Am J Physiol Lung Cell Mol Physiol 280: L165-L172, 2001

13. Basbaum C, Li D, Gensch E, Gallup M and Lemjabbar H: Mechanisms by which gram-positive bacteria and tobacco smoke stimulate mucin induction through the epidermal growth factor receptor (EGFR). Novartis Found Symp 248: 171-182, 2002.

14. Heijink IH, Brandenburg SM, Postma DS and van Oosterhout AJ: Cigarette smoke impairs airway epithelial barrier function and cell-cell contact recovery. Eur Respir J 39: 419-428, 2012.

15. Deshmukh HS, Case LM, Wesselkamper SC, et al: Metalloproteinases mediate mucin $5 \mathrm{AC}$ expression by epidermal growth factor receptor activation. Am J Respir Crit Care Med 171: 305-314, 2005

16. Cortijo J, Mata M, Milara J, et al: Aclidinium inhibits cholinergic and tobacco smoke-induced MUC5AC in human airways. Eur Respir J 37: 244-254, 2011.

17. Yu H, Li Q, Kolosov VP, Perelman JM and Zhou X: Regulation of cigarette smoke-mediated mucin expression by hypoxiainducible factor- $1 \alpha$ via epidermal grow th factor receptor-mediated signaling pathways. J Appl Toxicol 32: 282-292, 2012.

18. Thomas CM and Smart EJ: Caveolae structure and function. J Cell Mol Med 12: 796-809, 2008.

19. Stan RV: Structure of caveolae. Biochim Biophys Acta 1746 : 334-348, 2005

20. Hansen CG and Nichols BJ: Exploring the caves: Cavins, caveolins and caveolae. Trends Cell Biol 20: 177-186, 2010
21. Liu P, Rudick M and Anderson RG: Multiple functions of caveolin-1. J Biol Chem 277: 41295-41298, 2002.

22. Schwencke C, Braun-Dullaeus RC, Wunderlich C and Strasser RH Caveolae and caveolin in transmembrane signaling: Implications for human disease. Cardiovasc Res 70: 42-49, 2006.

23. Boscher C and Nabi IR: Caveolin-1: Role in cell signaling. Adv Exp Med Biol 729: 29-50, 2012.

24. Couet J, Sargiacomo M and Lisanti MP: Interaction of a receptor tyrosine kinase, EGF-R, with caveolins. Caveolin binding negatively regulates tyrosine and serine/threonine kinase activities. J Biol Chem 272: 30429-30438, 1997.

25. Gu D, Li H, Wang Z, Chen Q, Jiang J and Zhu H: Caveolin-1 inhibits the growth of human laryngeal squamous cell carcinoma and down regulates EGFR-MAPKs signaling pathway. Laryngoscope 117: 1782-1789, 2007.

26. Han F, Gu D, Chen Q and Zhu H: Caveolin-1 acts as a tumor suppressor by down-regulating epidermal growth factor receptormitogen-activated protein kinase signaling pathway in pancreatic carcinoma cell lines. Pancreas 38: 766-774, 2009.

27. Garrean S, Gao XP, Brovkovych V, et al: Caveolin-1 regulates NF-kappaB activation and lung inflammatory response to sepsis induced by lipopolysaccharide. J Immunol 177: 4853-4860, 2006.

28. Jin Y, Lee SJ, Minshall RD and Choi AM: Caveolin-1: A critical regulator of lung injury. Am J Physiol Lung Cell Mol Physiol 300: L151-L160, 2011

29. Yuan K, Huang C, Fox J, et al: Elevated inflammatory response in caveolin-1-deficient mice with Pseudomonas aeruginosa infection is mediated by STAT3 protein and nuclear factor kappaB (NF-kappaB). J Biol Chem 286: 21814-21825, 2011.

30. Kode A, Yang SR and Rahman I: Differential effects of cigarette smoke on oxidative stress and proinflammatory cytokine release in primary human airway epithelial cells and in a variety of transformed alveolar epithelial cells. Respir Res 7: 132, 2006

31. Yang SR, Chida AS, Bauter MR, et al: Cigarette smoke induces proinflammatory cytokine release by activation of NF-kappaB and posttranslational modifications of histone deacetylase in macrophages. Am J Physiol Lung Cell Mol Physiol 291: L46-57, 2006.

32. Lv XJ, Li YY, Zhang YJ, Mao M and Qian GS: Over-expression of caveolin-1 aggravate LPS-induced inflammatory response in AT-1 cells via up-regulation of cPLA2/p38 MAPK. Inflamm Res 59: 531-541, 2010.

33. Shao MX, Nakanaga T and Nadel JA: Cigarette smoke induces MUC5AC mucin overproduction via tumor necrosis factor-alphaconverting enzyme in human airway epithelial (NCI-H292) cells. Am J Physiol Lung Cell Mol Physiol 287: L420-L427, 2004.

34. Binker MG, Binker-Cosen AA, Richards D, Oliver B and Cosen-Binker LI: LPS-stimulated MUC5AC production involves Rac1-dependent MMP-9 secretion and activation in NCI-H292 cells. Biochem Biophys Res Commun 386: 124-129, 2009.

35. Yu H, Li Q, Kolosov VP, Perelman JM and Zhou X: Regulation of cigarette smoke-induced mucin expression by neuregulin1 $\beta /$ ErbB3 signalling in human airway epithelial cells. Basic Clin Pharmacol Toxicol 109: 63-72, 2011.

36. Chidlow JH Jr and Sessa WC: Caveolae, caveolins, and cavins: Complex control of cellular signalling and inflammation. Cardiovasc Res 86: 219-225, 2010.

37. Wang XM, Kim HP, Song R and Choi AM: Caveolin-1 confers antiinflammatory effects in murine macrophages via the MKK3/ p38 MAPK pathway. Am J Respir Cell Mol Biol 34: 434-442, 2006.

38. Hu G, Ye RD, Dinauer MC, Malik AB and Minshall RD: Neutrophil caveolin-1 expression contributes to mechanism of lung inflammation and injury. Am J Physiol Lung Cell Mol Physiol 294: L178-186, 2008

39. Park $\mathrm{JH}$ and Han HJ: Caveolin-1 plays important role in EGF-induced migration and proliferation of mouse embryonic stem cells: Involvement of PI3K/Akt and ERK. Am J Physiol Cell Physiol 297: C935-944, 2009

40. Takaguri A, Shirai H, Kimura K, et al: Caveolin-1 negatively regulates a metalloprotease-dependent epidermal growth factor receptor transactivation by angiotensin II. J Mol Cell Cardiol 50: 545-551, 2011.

41. Yang CM, Lee IT, Lin CC, et al: Cigarette smoke extract induces COX-2 expression via a PKCalpha/c-Src/EGFR, PDGFR/PI3K/ Akt/NF-kappaB pathway and p300 in tracheal smooth muscle cells. Am J Physiol Lung Cell Mol Physiol 297: L892-902, 2009.

42. Hegab AE, Sakamoto T, Nomura A, et al: Niflumic acid and AG-1478 reduce cigarette smoke-induced mucin synthesis: The role of hCLCA1. Chest 131: 1149-1156, 2007. 
43. Li N, Li Q, Zhou XD, Kolosov VP and Perelman JM: The effect of quercetin on human neutrophil elastase-induced mucin5AC expression in human airway epithelial cells. Int Immunopharmacol 14: 195-201, 2012.

44. Yang J, Yu HM, Zhou XD, Kolosov VP and Perelman JM: Study on TRPV1-mediated mechanism for the hypersecretion of mucus in respiratory inflammation. Mol Immunol 53: 161-171, 2013.

45. Wang $\mathrm{Y}$, Roche $\mathrm{O}, \mathrm{Xu} \mathrm{C}$, et al: Hypoxia promotes ligandindependent EGF receptor signaling via hypoxia-inducible factor-mediated upregulation of caveolin-1. Proc Natl Acad Sci USA 109: 4892-4897, 2012.

46. Hua H, Munk S and Whiteside CI: Endothelin-1 activates mesangial cell ERK1/2 via EGF-receptor transactivation and caveolin-1 interaction. Am J Physiol Renal Physiol 284: F303-F312, 2003.
47. Agelaki S, Spiliotaki M, Markomanolaki H, et al: Caveolin-1 regulates EGFR signaling in MCF-7 breast cancer cells and enhances gefitinib-induced tumor cell inhibition. Cancer Biol Ther 8: 1470-1477, 2009.

48. Mattsson CL, Andersson ER and Nedergaard J: Differential involvement of caveolin-1 in brown adipocyte signaling: Impaired beta3-adrenergic, but unaffected LPA, PDGF and EGF receptor signaling. Biochim Biophys Acta 1803: 983-989, 2010.

49. Sedding DG and Braun-Dullaeus RC: Caveolin-1: Dual role for proliferation of vascular smooth muscle cells. Trends Cardiovasc Med 16: 50-55, 2006. 